\title{
Efficacy and safety of apatinib treatment for advanced esophageal squamous cell carcinoma
}

This article was published in the following Dove Press journal:

OncoTargets and Therapy

7 August 2017

Number of times this article has been viewed

Jianqiang $\mathrm{Li}^{1}$

Lifen Wang ${ }^{2}$

'Department of Thoracic Oncological Surgery, ${ }^{2}$ Department of Medical Oncology, Zhejiang Cancer Hospital, Hangzhou, People's Republic of China
Objective: No standard chemotherapy is available for patients with advanced esophageal squamous cell carcinoma (ESCC) who have failed prior first-line chemotherapy. The aim of this study was to evaluate the efficacy and safety of apatinib, an oral VEGFR-2 inhibitor, as salvage treatment for advanced ESCC.

Patients and methods: After apatinib dosing, the efficacy and toxicity were evaluated in 62 patients with pretreated advanced ESCC from 2014 to 2016 at Zhejiang Cancer Hospital. In addition, survival analysis was performed by the Kaplan-Meier method.

Results: Among the 62 patients, 15 achieved partial response while 31 had stable disease with a response rate of $24.2 \%$ and a disease control rate of $74.2 \%$. Median progression-free survival (PFS) and overall survival were 115 and 209 days, respectively. Grade 3/4 toxicities $(59.7 \%)$ were acceptable. Patients with grade $3 / 4$ toxicities showed a longer PFS than those without (136 vs 63 days, $P=0.044$ ).

Conclusion: Apatinib is efficacious as second- or further-line treatment for advanced ESCC. Keywords: esophageal squamous cell carcinoma, apatinib, vascular endothelial growth factor, toxicity

\section{Introduction}

Esophageal carcinoma is one of the leading causes of cancer-related death worldwide, especially in Asia. ${ }^{1}$ Most Asian patients are diagnosed as having esophageal squamous cell carcinoma (ESCC), and the histology is somewhat different from non-Asian populations. ${ }^{2,3}$ Despite timely surgical interventions at an early stage, many cases tend to recur during the follow-ups. ${ }^{4,5}$ Currently, platinum-based regimens are a standard first-line treatment for advanced ESCC with a median progression-free survival (PFS) under 6 months. ${ }^{6}$ No definitive chemotherapeutic regimen has been properly established for those who have failed prior first-line chemotherapy.

Vascular endothelial growth factor (VEGF) could stimulate the growth of new blood vessels, regulate vascular permeability and exert anti-apoptotic effects in endothelial cells. It frequently becomes overexpressed in esophageal cancers. ${ }^{7,8}$ In addition, its overexpression was identified as a poor prognostic predictor for advanced ESCC. ${ }^{9}$

Previous studies have indicated that apatinib, a VEGFR-2 inhibitor, was potentially efficacious for solid carcinomas. ${ }^{10}$ As a small-molecule, VEGFR tyrosine kinase inhibitor improved PFS and overall survival (OS) in pretreated patients with advanced gastric cancer. ${ }^{11,12}$ However, no clinical studies have examined the efficacy and safety of apatinib treatment for advanced ESCC.

A retrospective study was conducted to evaluate the efficacy and safety of apatinib for advanced ESCC after failed prior first-/further-line treatment.
Correspondence: Lifen Wang

Department of Medical Oncology,

Zhejiang Cancer Hospital, East Banshan

Road, Hangzhou 310022, People's

Republic of China

Tel +86 57I 88I2 2082

Fax +86 57I 88I 22088

Email wanglifen1963@163.com 


\section{Patients and methods}

\section{Patient eligibility}

Patients with advanced ESCC receiving apatinib as second/ further-line treatment between March 2014 and June 2016 were included. All histological diagnoses of ESCC were made according to the histopathological criteria of WHO 2015 version. No local radiotherapy or interventional therapy was offered during apatinib dosing. The study protocol was approved by our institutional review board of Zhejiang Cancer Hospital. All participants provided informed consent prior to treatment.

\section{Treatment regimen}

Apatinib was administered at a daily dose of $500 \mathrm{mg}$, and one treatment cycle lasted 28 days. In addition, one dose reduction $(500-250 \mathrm{mg})$ was allowed for drug-related toxicity.

\section{Responses and toxicities}

Tumor efficacy was evaluated by the Response Evaluation Criteria in Solid Tumors (RECIST 1.1). Objective tumor responses included complete response (CR), partial response (PR), stable disease (SD) and progressive disease (PD). In addition, toxicities were assessed by the National Cancer Institute Common Toxicity Criteria version 4.0 (CTC 4.0). Tumor responses were evaluated for every two cycles when no noticeable sign of progression was present.

\section{Follow-ups and statistical analyses}

PFS denoted the time from the first dosing day of apatinib to documented progression or mortality from any cause. In addition, OS was defined as the time from the first dosing day to mortality or the last follow-up. Survival analysis was conducted using the Kaplan-Meier method and compared using log-rank test. The survival curves were plotted according to the Kaplan-Meier method. Statistical analysis was performed using SPSS version 17.0 (SPSS Inc., Chicago, IL, USA). The median follow-up period was 10.2 (2.0-22) months. Follow-ups were conducted up to October 30, 2016.

\section{Results}

\section{Patient characteristics}

A total of 62 patients diagnosed with ESCC were included in the current study. Among them, 54 were male and eight were female with a median age of 60.5 years. In addition, 46 of them were previous or current smokers and 16 belonged to never smoker category. All of them received platinum-based first-line chemotherapy. Apatinib was prescribed as secondline $(n=21)$ and further-line $(n=41)$ treatments. Performance status (PS) was $0-1$ in 52 patients and 2 in 10 patients. Patient characteristics are summarized in Table 1.
Table I Clinical characteristics of 62 patients

\begin{tabular}{ll}
\hline Variables & $\mathbf{N}(\%)$ \\
\hline Gender & \\
Male & $54(87.1)$ \\
Female & $8(12.9)$ \\
Age (years) & \\
$\quad$ Median (range) & $60.5(40-72)$ \\
$>60$ & $32(51.6)$ \\
$\leq 60$ & $30(48.4)$ \\
PS & \\
0-I & $52(83.9)$ \\
2 & $10(16.1)$ \\
Smoking history & \\
Yes & $46(74.2)$ \\
No & $16(25.8)$ \\
Alcohol use & \\
Yes & $49(79.0)$ \\
No & $13(21.0)$ \\
Location of tumor & \\
Upper third & $9(14.5)$ \\
Middle third & $24(38.7)$ \\
Lower third & $29(46.8)$ \\
Line of apatinib therapy & \\
Second & $21(33.9)$ \\
Further & $41(66.1)$ \\
Prior therapies in advanced stage & \\
Chemotherapy & $44(71.0)$ \\
Post-progression therapy after apatinib & $18(29.0)$ \\
Chemotherapy & \\
\hline Palliative treatment & $16(25.8)$ \\
A & $46(74.2)$ \\
\hline
\end{tabular}

Abbreviation: PS, performance status.

\section{Clinical efficacies}

The clinical responses were as follows: $\mathrm{CR}(\mathrm{n}=0), \mathrm{PR}(\mathrm{n}=15)$, $\mathrm{SD}(\mathrm{n}=31)$ and $\mathrm{PD}(\mathrm{n}=16)$. The values of objective response rate (ORR) and disease control rate (DCR) were $24.2 \%$ and $74.2 \%$, respectively. The median PFS was 115 days (95\% CI, 97-133; Figure 1), and the median OS was 209 days (95\% CI, 165-253; Figure 2).

No significant correlation existed in PFS among gender ( $P=0.51)$, age $(P=0.43)$, line of therapy $(P=0.43)$, smoking history $(P=0.23)$, location of tumor $(P=0.44)$ and PS $(P=0.06)$. Univariate analysis is detailed in Table 2. Patients with grade $3 / 4$ toxicities showed a longer PFS than those without grade $3 / 4$ toxicity ( 136 vs 63 days, $P=0.044$; Figure 3). Interestingly, PFS in individuals with grade $3 / 4$ hypertension and hand-foot syndrome was longer than that in other patients ( 153 vs 112 days, $P=0.037$ ).

Among 46 SD patients, 13 had a PFS of $>6$ months, and the patient characteristics are summarized in Table 3.

\section{Toxicity evaluations}

The median dose of apatinib was $500(250-500) \mathrm{mg}$. Ten dosage reductions were available. The rate of grade $3 / 4$ 


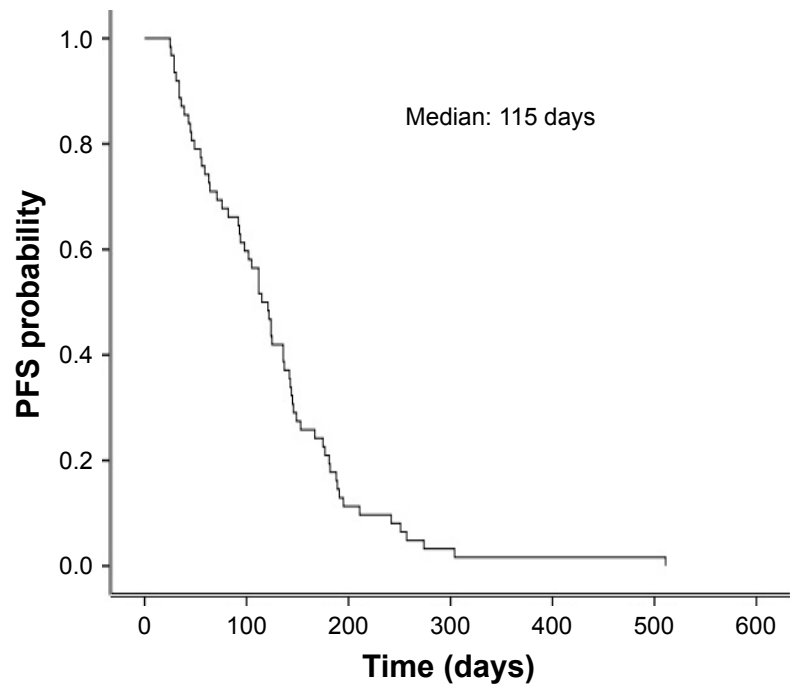

Figure I Kaplan-Meier curve of PFS after apatinib dosing. Abbreviation: PFS, progression-free survival.

toxicities was $59.7 \%(37 / 62)$. Five patients presented with grade 4 toxicity, including worsening proteinuria $(n=2)$, hypertension $(n=2)$ and hand-foot syndrome $(n=1)$. The most common grade 3/4 adverse events were as follows: hand-foot syndrome $(n=10)$, hypertension $(n=7)$, proteinuria $(n=7)$, hepatic injury $(n=5)$, fatigue $(n=3)$, esophagitis $(n=3)$ and nausea/vomiting $(\mathrm{n}=2$; Table 4$)$.

\section{Discussion}

To sum up, apatinib had some potential efficacy as a salvage treatment for advanced ESCC therapy. To the best of our knowledge, it represented the first-ever attempt of examining the efficacy and safety of apatinib for advanced ESCC.

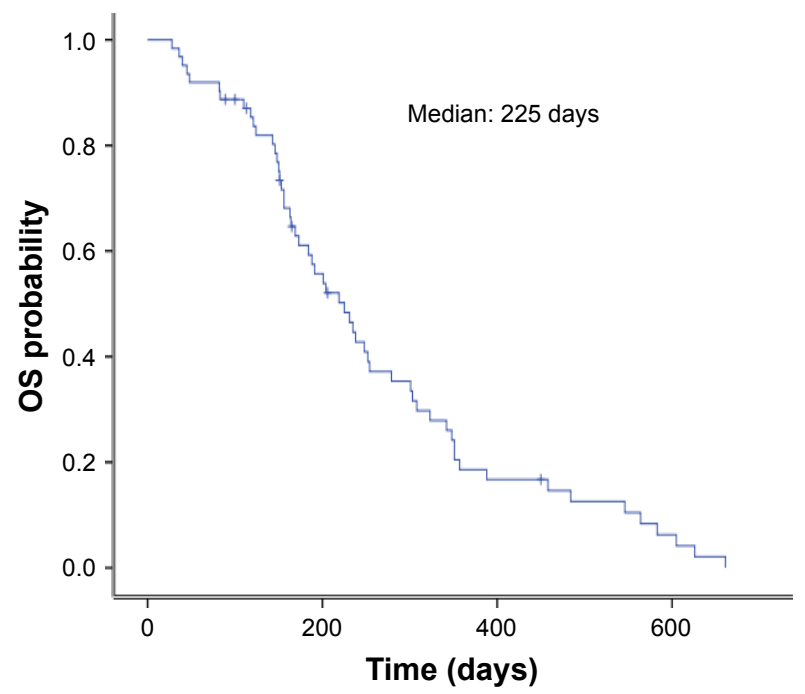

Figure 2 Kaplan-Meier curve of OS after apatinib dosing. Abbreviation: OS, overall survival.
Table 2 Univariate analysis of the current population $(n=62)$

\begin{tabular}{|c|c|c|c|c|c|c|}
\hline Characteristics & PFS & $95 \% \mathrm{Cl}$ & $P$-value & os & $95 \% \mathrm{Cl}$ & $P$-value \\
\hline Gender & & & $0.5 \mathrm{I}$ & & & 0.24 \\
\hline Male & 95 & $72-125$ & & 176 & $165-256$ & \\
\hline Female & 122 & $87-142$ & & 234 & 199-277 & \\
\hline Age (years) & & & 0.43 & & & 0.34 \\
\hline$>60$ & 110 & $107-133$ & & 231 & $168-266$ & \\
\hline$\leq 60$ & 117 & $87-121$ & & 201 & $176-254$ & \\
\hline PS & & & 0.06 & & & 0.04 \\
\hline $0-1$ & 132 & $106-157$ & & 254 & $226-312$ & \\
\hline 2 & 87 & $55-125$ & & 156 & $117-204$ & \\
\hline Line of therapy & & & 0.43 & & & 0.45 \\
\hline Second & 126 & $75-131$ & & 234 & $176-265$ & \\
\hline Further & III & $87-129$ & & 199 & $167-254$ & \\
\hline Smoking history & & & 0.23 & & & 0.55 \\
\hline Yes & 98 & $67-119$ & & 204 & $187-255$ & \\
\hline No & 124 & $89-132$ & & 255 & $211-269$ & \\
\hline Location of tumor & & & 0.44 & & & 0.25 \\
\hline $\begin{array}{l}\text { Upper and } \\
\text { middle third }\end{array}$ & 101 & $78-125$ & & 187 & $167-254$ & \\
\hline Lower third & 117 & $111-135$ & & 231 & $207-288$ & \\
\hline
\end{tabular}

Abbreviations: OS, overall survival; PFS, progression-free survival; PS, performance status.

Platinum-based agents are currently a standard first-line treatment for advanced ESCC, and the median PFS has a range of 4-6 months. ${ }^{13-15}$ Half of the patients unresponsive to first-line treatment might receive a second-line therapy. Yet, the median PFS remains at a range of 2-4 months. ${ }^{16,17}$ For patients who have failed second-line chemotherapy, no definitive chemotherapeutic regimen has been recommended. New treatment strategy is urgently needed for achieving a better PS.

Several studies have identified the blockage of VEGFR-2 as a promising therapy for inhibiting angiogenesis. ${ }^{18,19}$ Apatinib, the first oral VEGFR-2 inhibitor, has previously

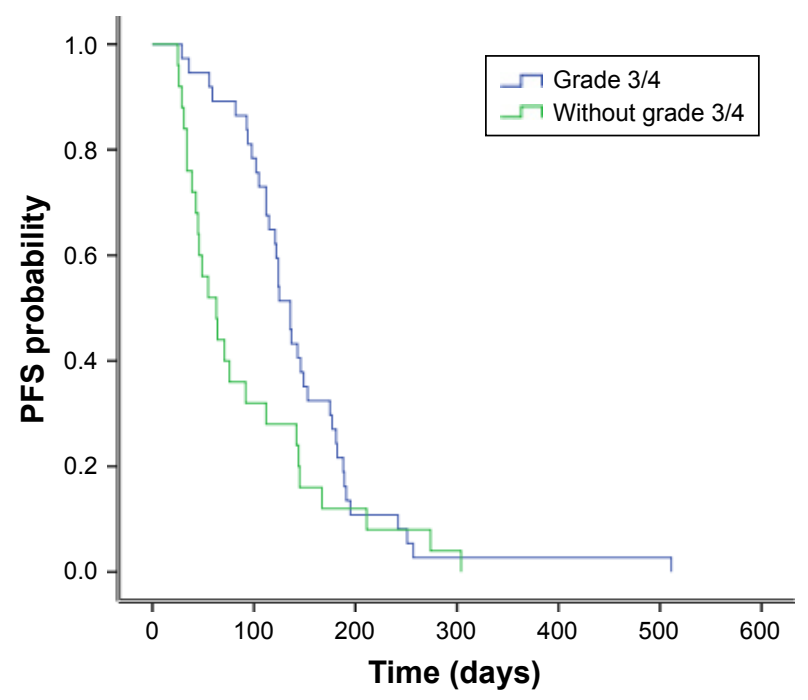

Figure 3 Comparison of PFS between patients with and without grade 3/4 toxicities. Abbreviation: PFS, progression-free survival. 
Table 3 Clinical profile of patients with PFS $>6$ months

\begin{tabular}{|c|c|c|c|c|c|c|c|}
\hline Case & Gender & Age (years) & Smoking & Line of therapy & Grade $3 / 4$ toxicity & PFS (days) & OS (days) \\
\hline I & Male & 56 & Yes & Second & Yes & 188 & 252 \\
\hline 2 & Male & 63 & Yes & Third & Yes & 181 & 254 \\
\hline 3 & Male & 64 & No & Second & Yes & 195 & $357+$ \\
\hline 4 & Female & 54 & No & Fifth & No & 251 & 661 \\
\hline 5 & Male & 65 & Yes & Second & Yes & 304 & 564 \\
\hline 6 & Female & 48 & No & Fifth & No & 189 & 216 \\
\hline 7 & Male & 63 & Yes & Third & Yes & 242 & 458 \\
\hline 8 & Male & 64 & No & Second & Yes & 511 & 583 \\
\hline 9 & Female & 62 & No & Second & Yes & 251 & 279 \\
\hline 10 & Female & 54 & No & Third & No & 274 & 348 \\
\hline II & Male & 57 & Yes & Third & Yes & 191 & 303 \\
\hline 12 & Male & 54 & Yes & Second & No & 182 & 191 \\
\hline 13 & Male & 50 & Yes & Fourth & No & 211 & 351 \\
\hline
\end{tabular}

Abbreviations: OS, overall survival; PFS, progression-free survival.

demonstrated survival benefits for metastatic gastric cancer. ${ }^{12}$ Although approved domestically for gastric cancer treatment, apatinib was also effective for patients with advanced breast carcinoma and lung cancer who are unresponsive to standard pretreatment. ${ }^{20,21}$ In the current study, the values of DCR and ORR were $74.2 \%$ and $24.2 \%$, respectively. There was a trend of better efficacy compared with secondline chemotherapy for advanced ESCC. ${ }^{5}$ Interestingly, patients with grade $3 / 4$ toxicities had a longer PFS than those without grade 3/4 toxicities. Patients with hypertension and hand-foot syndrome benefited more than those with other adverse events. Together with previous study, ${ }^{12}$ our results indicated that some toxicities would be predictive factors for the efficacy of apatinib treatment.

Hand-foot skin reaction, proteinuria and hypertension were the most common adverse events in apatinib treatment, with grade $3 / 4$ adverse events occurring in over $60 \%$ of patients with gastric carcinoma. ${ }^{11,12}$ Over $20 \%$ of patients experienced dose modifications with a recommended daily dose of apatinib $(850 \mathrm{mg})$ treatment in a Phase III trial. ${ }^{12}$ In another trial, the recommended daily dose was $500 \mathrm{mg}$ and grade $3 / 4$ toxicities significantly decreased, and the efficacy was similar to those of high dose for breast carcinoma. ${ }^{20}$ In the current study, a recommended dose of $500 \mathrm{mg}$ was used. The results showed that grade $3 / 4$ toxicities occurred in over half of the patients. Although different daily apatinib doses were used for gastric carcinoma $(850 \mathrm{mg})$ and breast carcinoma (500 mg), similar toxicities were observed. It was considered that several patients with gastric carcinoma underwent previous gastrectomy, and the absorption ability of apatinib might be lowered.

Retrospective nature and a small sample size were two major limitations of the current study. In addition, the dose of $500 \mathrm{mg}$ apatinib adopted in this study was not widely recommended. Hence, this dose must be confirmed by further prospective studies. However, without prospective clinical studies in the literature, our study may be deemed as meaningful.

\section{Conclusion}

Our results support that apatinib is efficacious for advanced ESCC as salvage treatment. However, further prospective studies are required to fully elucidate its efficacy and toxicity.

Table 4 Major toxicities of apatinib dosing

\begin{tabular}{|c|c|c|c|c|}
\hline Toxicity & Total (\%) & Grades 3/4 (\%) & Dosage reduction (\%) & Discontinuation (\%) \\
\hline Hand-foot syndrome & $32(51.6)$ & $10(16.1)$ & $2(20.0)$ & I (33.3) \\
\hline Hypertension & $13(21.0)$ & $7(11.3)$ & $2(20.0)$ & I (33.3) \\
\hline Proteinuria & $15(24.2)$ & $7(11.3)$ & $2(20.0)$ & I (33.3) \\
\hline Hepatic injury & $12(19.4)$ & $5(8.1)$ & $0(0.0)$ & $0(0.0)$ \\
\hline Fatigue & $9(14.5)$ & $3(4.8)$ & $2(20.0)$ & $0(0.0)$ \\
\hline Esophagitis & $4(6.5)$ & $3(4.8)$ & $2(20.0)$ & $0(0.0)$ \\
\hline Nausea/vomiting & $9(14.5)$ & $2(4.8)$ & $0(0.0)$ & $0(0.0)$ \\
\hline
\end{tabular}




\section{Disclosure}

The authors report no conflicts of interest in this work.

\section{References}

1. Zheng R, Zeng H, Zuo T, et al. Lung cancer incidence and mortality in China, 2011. Thorac Cancer. 2016;7(1):94-99.

2. Zhang Y. Epidemiology of esophageal cancer. World J Gastroenterol. 2013;19(34):5598-5606.

3. Low DE. Evolution in surgical management of esophageal cancer. Dig Dis. 2013;31(1):21-29.

4. Song ZB, Lin BC, Li B, et al. Preoperative elevation of serum C-reactive protein as an indicator of poor prognosis for early-stage esophageal squamous cell carcinoma. Kaohsiung J Med Sci. 2013;29(12): 662-666.

5. Song Z, Zhang Y. Second-line docetaxel-based chemotherapy after failure of fluorouracil-based first-line treatment for advanced esophageal squamous cell carcinoma. Onco Targets Ther. 2014;7:1875-1881.

6. Hamamoto Y, Kitagawa Y. Current perspective of treatment for advanced esophageal squamous cell carcinoma. Nihon Shokakibyo Gakkai Zasshi. 2014;111(2):253-259.

7. Qu W, Fu JD, Yang F, et al. Clinical implications of PTEN and VEGF expression status, as well as microvessel density in esophageal squamous cell carcinoma. Oncol Lett. 2015;10(3):1409-1415.

8. Shih CH, Ozawa S, Ando N, Ueda M, Kitajima M. Vascular endothelial growth factor expression predicts outcome and lymph node metastasis in squamous cell carcinoma of the esophagus. Clin Cancer Res. 2000; 6(3):1161-1168.

9. Yang PW, Hsieh MS, Huang YC, Hsieh CY, Chiang TH, Lee JM. Genetic variants of EGF and VEGF predict prognosis of patients with advanced esophageal squamous cell carcinoma. PLoS One. 2014;9(6): e100326.

10. Ding J, Chen X, Gao Z, et al. Metabolism and pharmacokinetics of novel selective vascular endothelial growth factor receptor-2 inhibitor apatinib in humans. Drug Metab Dispos. 2013;41:1195-1210.

11. Li J, Qin S, Xu J, et al. Apatinib for chemotherapy-refractory advanced metastatic gastric cancer: results from a randomized, placebocontrolled, parallel-arm, phase II trial. J Clin Oncol. 2013;31(26): 3219-3225.
12. Li J, Qin S, Xu J, et al. Randomized, double-blind, placebo-controlled phase III trial of apatinib in patients with chemotherapy-refractory advanced or metastatic adenocarcinoma of the stomach or gastroesophageal junction. J Clin Oncol. 2016;34:1448-1454.

13. Chen MQ, Chen C, Lu HJ, Xu BH. The efficacy and toxicities of combined lobaplatin with paclitaxel as a first-line chemotherapy for advanced esophageal squamous cell carcinoma. J Thorac Dis. 2015;7(10): 1749-1755.

14. Lee SJ, Kim S, Kim M, et al. Capecitabine in combination with either cisplatin or weekly paclitaxel as a first-line treatment for metastatic esophageal squamous cell carcinoma: a randomized phase II study. BMC Cancer. 2015;15:693.

15. Shi Y, Qin R, Wang ZK, Dai GH. Nanoparticle albumin-bound paclitaxel combined with cisplatin as the first-line treatment for metastatic esophageal squamous cell carcinoma. Onco Targets Ther. 2013;6: 585-591.

16. Tian J, Shang M, Shi SB, Han Y, Xu J. Cetuximab plus pemetrexed as second-line therapy for fluorouracil-based pre-treated metastatic esophageal squamous cell carcinoma. Cancer Chemother Pharmacol. 2015;76(4):829-834.

17. Li X, Lin W, Wang H, Lin W, Lin S, Lin Y. Phase II trial of second-line chemotherapy with docetaxel and capecitabine in advanced esophageal squamous cell carcinoma. Med Oncol. 2013;30(4):746.

18. Aziz MA, Serya RA, Lasheen DS, et al. Discovery of potent VEGFR-2 inhibitors based on furopyrimidine and thienopyrimidne scaffolds as cancer targeting agents. Sci Rep. 2016;6:24460.

19. Ghavamipour F, Shahangian SS, Sajedi RH, Arab SS, Mansouri K, Aghamaali MR. Development of a highly-potent anti-angiogenic VEGF8-109 heterodimer by directed blocking of its VEGFR-2 binding site. FEBS J. 2014;281(19):4479-4494.

20. Hu X, Zhang J, Xu B, et al. Multicenter phase II study of apatinib, a novel VEGFR inhibitor in heavily pretreated patients with metastatic triple-negative breast cancer. Int J Cancer. 2014;135(8):1961-1969.

21. Song Z, Yu X, Lou G, Shi X, Zhang Y. Salvage treatment with apatinib for advanced non-small-cell lung cancer. Onco Targets Ther. 2017;10: $1821-1825$
OncoTargets and Therapy

\section{Publish your work in this journal}

OncoTargets and Therapy is an international, peer-reviewed, open access journal focusing on the pathological basis of all cancers, potential targets for therapy and treatment protocols employed to improve the management of cancer patients. The journal also focuses on the impact of management programs and new therapeutic agents and protocols on

\section{Dovepress}

patient perspectives such as quality of life, adherence and satisfaction. The manuscript management system is completely online and includes a very quick and fair peer-review system, which is all easy to use. Visit http://www.dovepress.com/testimonials.php to read real quotes from published authors. 